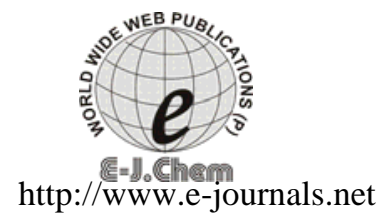

ISSN: 0973-4945; CODEN ECJHAO

E-Journal of Chemistry

2011, 8(S1), S330-S334

\title{
Theoretical Prediction of Ultrasonic Velocity in Binary Liquid Mixtures
}

\author{
SHAIK.BABU \\ Basic Engineering Department \\ Chalapathi Institute of Engineering and Technology \\ Guntur, Andhra Pradesh, India \\ babu.computers@gmail.com
}

Received 29 January 2011; Accepted 12 April 2011

\begin{abstract}
Theoretical values of ultrasonic velocity in the binary mixtures of chlorobenzene with benzene have been evaluated at different temperatures from 303.15 K-323.15 K using Nomoto's relation, ideal mixture relation, Junjie's method, collision factor theory and free length theory. Theoretical values were compared with the experimental values and the validity of the theories was checked by applying the chi-square test for goodness of fit and by calculating the average percentage error (APE).
\end{abstract}

Keywords: Ultrasonic velocity, Binary mixtures, Theoretical prediction

\section{Introduction}

Measurement of ultrasonic velocity gives the valuable information about the physicochemical behavior of the liquid and liquid mixtures. Several relations, semi-empirical formula and theories are available for the theoretical computation of ultrasonic velocity in liquid and liquid mixtures $^{1-5}$. Further, the best suitable theory for the given molecular system under study is also picked out by calculating the average percentage error and chi-square test.

\section{Experimental}

The ultrasonic velocity was measured at different temperatures from $303.15 \mathrm{~K}-323.15 \mathrm{~K}$ using a single crystal interferometer with a high degree of accuracy operating at a frequency of $2 \mathrm{MHz}$. The density was measured at different temperatures from $303.15 \mathrm{~K}-323.15 \mathrm{~K}$ using specific gravity bottle method by the standard procedure.

\section{Theory}

The following are relations/theories used for the prediction of ultrasonic velocity in the binary liquid mixtures. 
Nomoto’s relations (NOM)

Where, Molar sound velocity,

$$
\begin{aligned}
\mathrm{U}_{\mathrm{NOM}} & =\left[\frac{X_{1} R_{1}+X_{2} R_{2}}{X_{1} V_{1}+X_{2} V_{2}}\right]^{5} \\
R_{1} & =\frac{m_{1}}{d_{1}} U_{1}^{1 / 2} ; \quad R_{\mathrm{z}}=\frac{m_{\mathrm{z}}}{d_{\mathrm{z}}} U_{\mathrm{z}}^{1 / 6} \\
V_{1} & =\frac{m_{1}}{d_{1}} ; V_{2}=\frac{m_{\mathrm{z}}}{d_{\mathrm{z}}}
\end{aligned}
$$

Molar volume

$$
\mathrm{U}_{\mathrm{IMR}}=\left[\frac{1}{X_{1} m_{1}+X_{2} m_{2}}\right]^{1 / 2}\left[\frac{X_{2}}{m_{1} U_{1}^{2}}+\frac{X_{\mathrm{g}}}{m_{2} U_{2}^{2}}\right]^{-1 / 2}
$$

Junjie's method(JM)

Collision factor theory (CFT)

$$
\mathrm{U}_{\mathrm{JM}}=\left[\frac{X_{2} V_{2}+X_{2} V_{\mathrm{g}}}{\left(X_{2} m_{1}+X_{2} m_{2}\right)^{\frac{1}{2}}}\right]\left[\frac{X_{1} V_{1}}{d_{1} U_{1}^{2}}+\frac{X_{2} V_{2}}{d_{2} U_{2}^{2}}\right]^{-1 / 2}
$$

$$
\mathrm{U}_{\mathrm{CFT}}=U_{\infty}\left[\left(X_{4} 1 S_{4} 1+X_{4} 2 S_{4} 2\right)\left(X_{4} 1 B_{4} 1+X_{4} 2 B_{4} 2\right) / V_{4} m\right]
$$

Where Molar volume of mixture,

$$
V_{m}=\frac{X_{1} m_{1}+X_{2} m_{2}}{d_{\exp }}
$$

Actual Volume,

$$
\mathrm{B}=\frac{4}{3} \pi r^{\mathrm{2}} N
$$

Molecular radius,

$$
r=\left[\frac{3 b}{16 \pi N}\right]^{\frac{1}{3}}
$$

Vander Waal's constant,

$$
b=\frac{m}{d}\left[1-\frac{R T}{m U^{2}}\left(\frac{\sqrt{1+m U^{2}}}{3 R T}-1\right)\right]
$$

Temperature dependent constant, $\mathrm{U}_{\infty}=1600 \mathrm{~m} / \mathrm{sec}, N$ is the Avagadro's number.

Free length theory (FLT)

Where,

$$
\begin{aligned}
& \mathrm{U}_{\mathrm{CFT}}=\frac{K}{L_{f_{m t x}} d_{\exp }^{1 / 2}} \\
& E_{\operatorname{mix}}=2\left[\frac{V_{m}-\left(X_{1} V_{01}+X_{2} V_{02}\right)}{X_{1} Y_{1}+X_{2} Y_{2}}\right]
\end{aligned}
$$

Molar volume at absolute zero, $V_{01}=V_{1} \frac{U_{1}}{U_{x z}} ; V_{02}=V_{2} \frac{U_{2}}{U_{\mathrm{ar}}}$

Surface area per mole,

$$
Y_{1}=\frac{2\left(V_{1}-V_{01}\right)}{L_{f_{1}}} ; \quad Y_{2}=\frac{2\left(V_{2}-V_{02}\right)}{L_{f_{2}}}
$$

Where, 1, 2, represents the first and second component of the liquid mixture and the other symbols have their usual meanings.

\section{Results and Discussion}

The experimental values along with the values calculated theoretically using Nomoto's relation, collision factor theory, free length theory, ideal mixture relation and Junjie's method for the system chlorobenzene + benzene at different temperatures $303.15 \mathrm{~K}-323.15 \mathrm{~K}$ 
are given in Table 1 . The validity of the theories was checked by applying Chi-square test and by calculating average percentage error.

\section{Chi-square test for goodness of fit}

According to Karl Pearson ${ }^{6}$, the Chi-square value is calculated using the formula,

$$
\left(x^{2}\right)=\sum_{i=1}^{n} \frac{\left.\left(U_{m i x(a b s)}-U_{m i x f c a t}\right)\right)^{2}}{U_{m i x(c a t)}}
$$

For $(n-1)$ degrees of freedom, where, $\mathrm{n}$ is the number of data used.

\section{Average percentage error (APE)}

The average percentage Error $^{7}$ is calculated using the relation,

$$
A P E=\frac{1}{n} \sum \frac{\left(U_{\text {mixtabsi }}-U_{\text {mixtcan }}\right)}{U_{\text {mixtoss }}} X 100 \%
$$

Where, $n$ - number of data used. Umix(obs) = experimental values of ultrasonic velocities. $\operatorname{Umix}(\mathrm{cal})=$ computed values of ultrasonic velocities.

It can be seen from Table 1 that the theoretical values of ultrasonic velocity computed by various theories show deviation from experimental values. The reason may be the limitations and approximations incorporated in these theories.

Table 1. Experimental and computed values of Ultrasonic velocity for chlorobenzene + benzene system at $303.15 \mathrm{~K}-323.15 \mathrm{~K}$

\begin{tabular}{ccccccc}
\hline $\mathrm{X}_{1}$ & $\begin{array}{c}\mathrm{U}_{\text {exp }} \\
\mathrm{ms}^{-1}\end{array}$ & $\begin{array}{c}\mathrm{U}_{\mathrm{NOM}} \\
\mathrm{ms}^{-1}\end{array}$ & $\begin{array}{c}\mathrm{U}_{\text {IMR }} \\
\mathrm{ms}^{-1}\end{array}$ & $\begin{array}{c}\mathrm{U}_{\mathrm{JM}} \\
\mathrm{ms}^{-1}\end{array}$ & $\begin{array}{c}\mathrm{U}_{\mathrm{CFT}} \\
\mathrm{ms}^{-1}\end{array}$ & $\begin{array}{c}\mathrm{U}_{\text {FLT }} \\
\mathrm{ms}^{-1}\end{array}$ \\
\hline 0.0000 & 1281.21 & 1281.21 & 1281.21 & 1281.21 & 1281.21 & 1281.21 \\
0.0887 & 1275.93 & 1278.01 & 1272.21 & 1275.43 & 1263.67 & 1212.05 \\
0.1796 & 1271.26 & 1274.81 & 1264.37 & 1270.28 & 1247.96 & 1157.49 \\
0.2728 & 1267.16 & 1271.61 & 1257.72 & 1265.73 & 1234.53 & 1116.27 \\
0.3686 & 1263.59 & 1268.43 & 1252.28 & 1261.75 & 1223.83 & 1087.73 \\
0.4668 & 1260.50 & 1265.24 & 1248.12 & 1258.35 & 1216.38 & 1071.86 \\
0.5677 & 1257.83 & 1262.07 & 1245.30 & 1255.49 & 1212.74 & 1069.35 \\
0.6714 & 1255.48 & 1258.89 & 1243.89 & 1253.18 & 1213.52 & 1081.79 \\
0.7779 & 1253.38 & 1255.73 & 1244.02 & 1251.40 & 1219.39 & 1112.06 \\
0.8874 & 1251.40 & 1252.57 & 1245.80 & 1250.14 & 1231.08 & 1165.12 \\
1.0000 & 1249.41 & 1249.41 & 1249.41 & 1249.41 & 1249.41 & 1249.41 \\
& APE & -0.2220 & 0.5963 & 0.1064 & 2.1129 & 9.2373 \\
& Chi Square fit & 0.0940 & 0.6738 & 0.0219 & 8.7186 & 182.0335 \\
0.0000 & 1258.23 & 1258.23 & 1258.23 & 1258.23 & 1258.23 & 1258.23 \\
0.0887 & 1253.24 & 1255.48 & 1249.65 & 1252.87 & 1241.35 & 1195.06 \\
0.1796 & 1248.88 & 1252.73 & 1242.23 & 1248.14 & 1226.27 & 1144.72 \\
0.2728 & 1245.12 & 1249.99 & 1235.99 & 1244.01 & 1213.42 & 1106.37 \\
0.3686 & 1241.93 & 1247.24 & 1230.98 & 1240.47 & 1203.26 & 1079.63 \\
0.4668 & 1239.24 & 1244.51 & 1227.24 & 1237.49 & 1196.30 & 1064.65 \\
0.5677 & 1237.01 & 1241.77 & 1224.84 & 1235.08 & 1193.10 & 1062.19 \\
0.6714 & 1235.15 & 1239.04 & 1223.88 & 1233.22 & 1194.25 & 1073.81 \\
\hline \multicolumn{7}{c}{$308.15 \mathrm{~K}$} \\
\hline
\end{tabular}


Theoretical Prediction of Ultrasonic Velocity in Binary Liquid Mixtures

S333

\begin{tabular}{|c|c|c|c|c|c|c|}
\hline 0.7779 & 1233.59 & 1236.31 & 1224.47 & 1231.90 & 1200.43 & 1102.24 \\
\hline 0.8874 & 1232.20 & 1233.59 & 1226.74 & 1231.12 & 1212.37 & 1152.03 \\
\hline \multirow[t]{4}{*}{1.0000} & 1230.87 & 1230.87 & 1230.87 & 1230.87 & 1230.87 & 1230.87 \\
\hline & APE & -0.2511 & 0.5883 & 0.0883 & 2.0915 & 8.6826 \\
\hline & Chi Square fit & 0.1177 & 0.6450 & 0.0150 & 8.4000 & 156.9854 \\
\hline & \multicolumn{6}{|c|}{$313.15 \mathrm{~K}$} \\
\hline 0.0000 & 1235.39 & 1235.39 & 1235.39 & 1235.39 & 1235.39 & 1235.39 \\
\hline 0.0887 & 1230.70 & 1233.10 & 1227.24 & 1230.47 & 1219.19 & 1177.56 \\
\hline 0.1796 & 1226.67 & 1230.82 & 1220.25 & 1226.17 & 1204.76 & 1131.08 \\
\hline 0.2728 & 1223.26 & 1228.54 & 1214.43 & 1222.48 & 1192.52 & 1095.42 \\
\hline 0.3686 & 1220.45 & 1226.26 & 1209.85 & 1219.37 & 1182.93 & 1070.43 \\
\hline 0.4668 & 1218.19 & 1223.98 & 1206.54 & 1216.85 & 1176.49 & 1056.38 \\
\hline 0.5677 & 1216.42 & 1221.71 & 1204.60 & 1214.89 & 1173.74 & 1054.07 \\
\hline 0.6714 & 1215.07 & 1219.44 & 1204.10 & 1213.50 & 1175.29 & 1065.04 \\
\hline 0.7779 & 1214.06 & 1217.17 & 1205.17 & 1212.66 & 1181.79 & 1091.84 \\
\hline 0.8874 & 1213.29 & 1214.90 & 1207.95 & 1212.37 & 1193.97 & 1138.71 \\
\hline \multirow[t]{3}{*}{1.0000} & 1212.63 & 1212.63 & 1212.63 & 1212.63 & 1212.63 & 1212.63 \\
\hline & APE & -0.2816 & 0.5808 & 0.0696 & 2.0663 & 8.1749 \\
\hline & Chi Square fit & 0.1449 & $\begin{array}{l}0.6182 \\
318.15 \mathrm{~K}\end{array}$ & 0.0094 & 8.0597 & 135.9120 \\
\hline 0.0000 & 1212.58 & 1212.58 & 1212.58 & 1212.58 & 1212.58 & 1212.58 \\
\hline 0.0887 & 1208.58 & 1210.73 & 1204.84 & 1208.05 & 1197.02 & 1159.47 \\
\hline 0.1796 & 1205.18 & 1208.88 & 1198.24 & 1204.16 & 1183.21 & 1116.48 \\
\hline 0.2728 & 1202.36 & 1207.03 & 1192.83 & 1200.87 & 1171.55 & 1083.32 \\
\hline 0.3686 & 1200.08 & 1205.19 & 1188.65 & 1198.18 & 1162.49 & 1059.98 \\
\hline 0.4668 & 1198.29 & 1203.34 & 1185.75 & 1196.07 & 1156.54 & 1046.84 \\
\hline 0.5677 & 1196.94 & 1201.49 & 1184.22 & 1194.54 & 1154.22 & 1044.73 \\
\hline 0.6714 & 1195.94 & 1199.65 & 1184.15 & 1193.58 & 1156.13 & 1055.13 \\
\hline 0.7779 & 1195.21 & 1197.80 & 1185.67 & 1193.19 & 1162.93 & 1080.48 \\
\hline 0.8874 & 1194.65 & 1195.96 & 1188.93 & 1193.37 & 1175.32 & 1124.68 \\
\hline \multirow[t]{3}{*}{1.0000} & 1194.12 & 1194.12 & 1194.12 & 1194.12 & 1194.12 & 1194.12 \\
\hline & APE & -0.2487 & 0.6357 & 0.1152 & 2.1040 & 7.7713 \\
\hline & Chi Square fit & 0.1118 & $\begin{array}{l}0.7287 \\
323.15 \mathrm{~K}\end{array}$ & 0.0243 & 8.2183 & 120.1387 \\
\hline 0.0000 & 1189.91 & 1189.91 & 1189.91 & 1189.91 & 1189.91 & 1189.91 \\
\hline 0.0887 & 1186.42 & 1188.49 & 1182.56 & 1185.77 & 1174.96 & 1140.94 \\
\hline 0.1796 & 1183.51 & 1187.06 & 1176.37 & 1182.26 & 1161.73 & 1101.07 \\
\hline 0.2728 & 1181.17 & 1185.64 & 1171.35 & 1179.36 & 1150.62 & 1070.17 \\
\hline 0.3686 & 1179.34 & 1184.21 & 1167.56 & 1177.07 & 1142.07 & 1048.37 \\
\hline 0.4668 & 1177.98 & 1182.79 & 1165.06 & 1175.37 & 1136.59 & 1036.10 \\
\hline 0.5677 & 1177.04 & 1181.37 & 1163.94 & 1174.26 & 1134.69 & 1034.22 \\
\hline 0.6714 & 1176.43 & 1179.94 & 1164.29 & 1173.73 & 1136.97 & 1044.18 \\
\hline 0.7779 & 1176.08 & 1178.52 & 1166.25 & 1173.79 & 1144.07 & 1068.28 \\
\hline 0.8874 & 1175.86 & 1177.09 & 1169.98 & 1174.44 & 1156.71 & 1110.15 \\
\hline \multirow[t]{3}{*}{1.0000} & 1175.67 & 1175.67 & 1175.67 & 1175.67 & 1175.67 & 1175.67 \\
\hline & APE & -0.2409 & 0.6662 & 0.1369 & 2.1219 & 7.3990 \\
\hline & Chi Square fit & 0.1033 & 0.7868 & 0.0336 & 8.2175 & 106.5289 \\
\hline
\end{tabular}


Table 1 shows that the Chi-square value and APE value are minimum for Nomoto's relation than those obtained by other theories. However for the binary mixtures of chlorobenzene + benzene these values are minimum for JM. When two liquids are mixed, the interaction between the molecules of the two liquids takes place because of the presence of various forces like dispersive force, charge transfer, hydrogen bonding dipole-dipole and dipole-induced dipole interactions. Hence the observed deviation shows that the molecular interaction is taking place between the unlike molecules in the liquid mixture ${ }^{8}$. Similar kinds of results were obtained by earlier workers ${ }^{9-11}$.

\section{Conclusion}

Ultrasonic velocities predicted using NOM, IMR, JM, CFT and FLT were compared with experimentally measured velocity values at different temperatures from $303.15 \mathrm{~K}-323.15 \mathrm{~K}$ for the binary mixture of chlorobenzene+benzene. It may be concluded that nomoto's relation is best suited for the binary mixtures of chlorobenzene+benzene at all the temperatures. The observed deviation of theoretical values of velocity from the experimental values is attributed to the presence of intermolecular interactions in the system studied.

\section{References}

1. $\quad$ Nomoto O, J Phys Soc Jpn., 1958, 13,1528.

2. Schaafs W, Molekulara Kustik, Springer, Verlag, Berlin-Gottinger-Heidelberg, 1963, Chapters 11 \& 12 .

3. Jacobson B, Acta Chem Scand, 1952, 6, 1485.

4. Van Dael W and Vangeel E, Proc Int Conf on calorimetry and thermodynamics, Warasa, 1955, 555.

5. Junjie Z, J China Univ Sci Tech., 1984, 14, 298.

6. Pearson K, Fundamentals of Mathematical Statistics, Eds., Gupta S G and Kapoor V K, S Chand and Company, New Delhi, India, 1973, 903.

7. Jyh-Shing and Roger Jang, IEEE Transactions on Systems, Man and Cybernetics, 1993, 23(4), 665-685.

8. Rama Rao G V, Viswanatha Sarma A, Siva Rama Krishna J and Rambabu C, Indian J Pure Appl Phys., 2005, 43, 345-354.

9. Uvarani R and Punitha S, E-J Chem., 2009, 6(S1), S235-S238.

10. Sumathi T and Abeetha K, E-J Chem., 2011, 8(1), 252-256.

11. Vasantharani P, Muthu shailaja S, Kannappan A N, Ezhil Pavai R, J Appl Sci., 2008, 8(12), 2329-2332. 


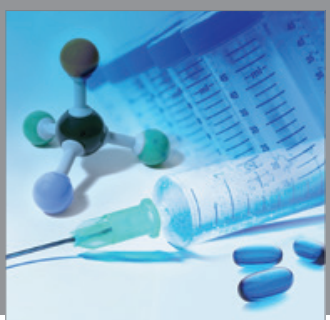

International Journal of

Medicinal Chemistry

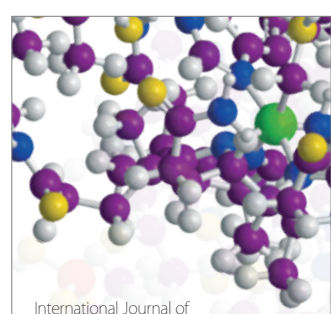

Carbohydrate Chemistry

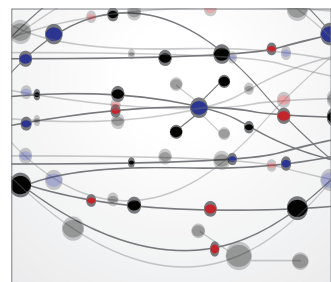

The Scientific World Journal
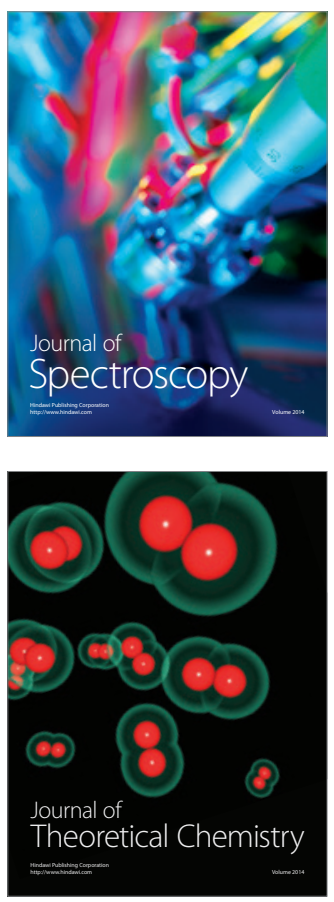
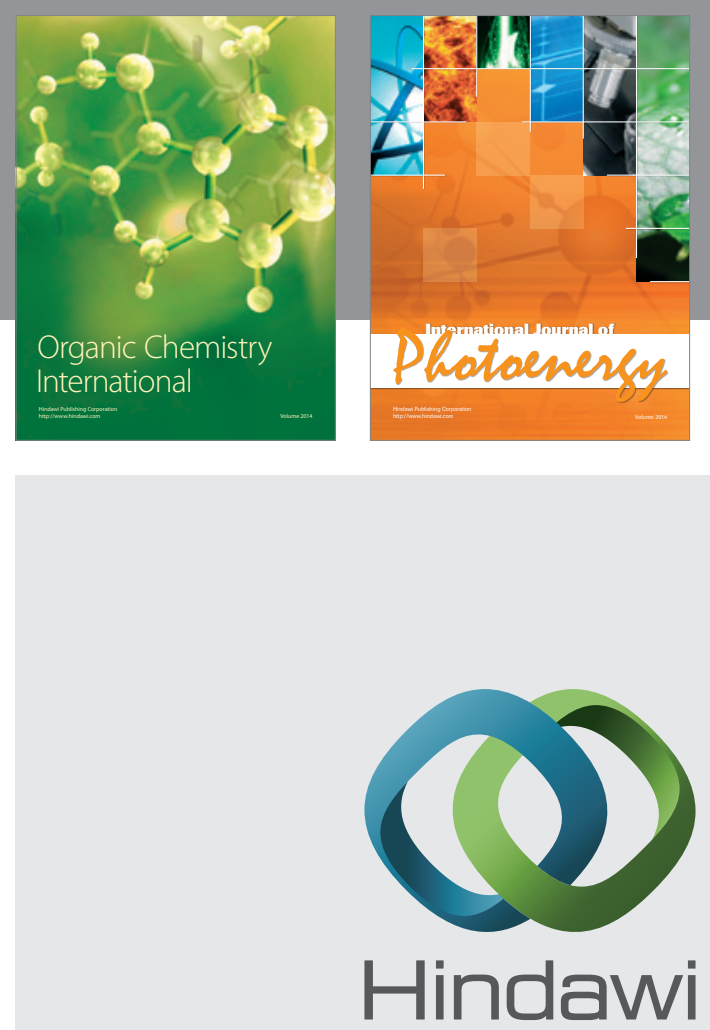

Submit your manuscripts at

http://www.hindawi.com
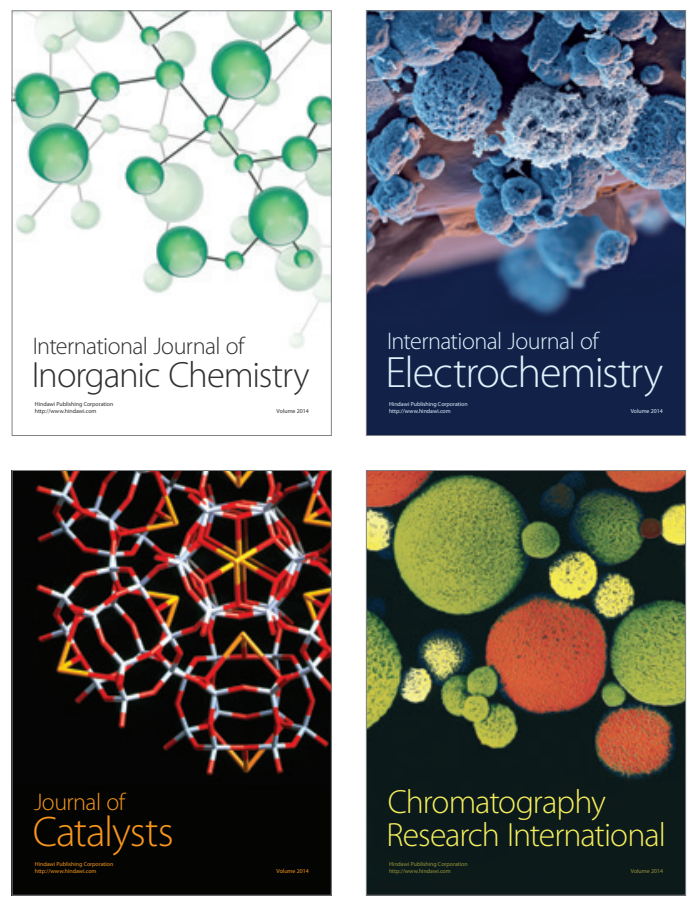
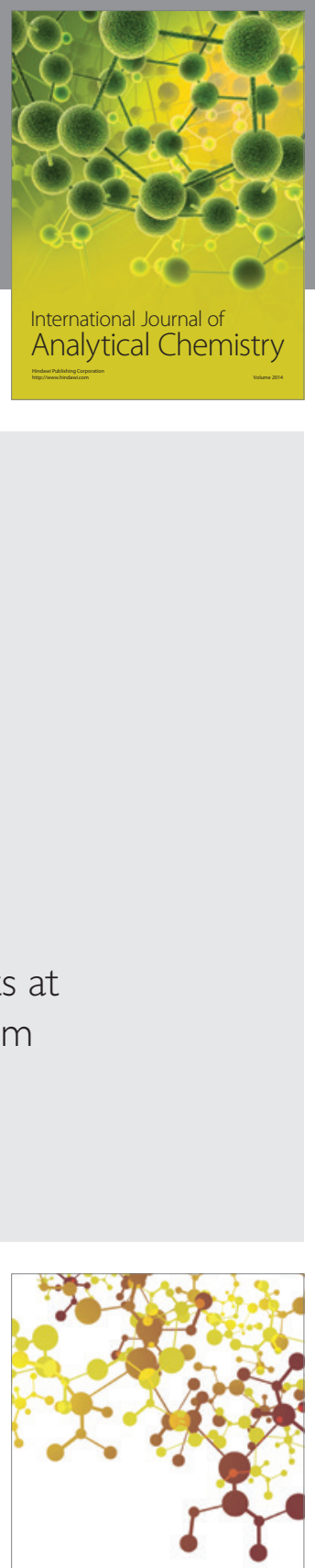

Journal of

Applied Chemistry
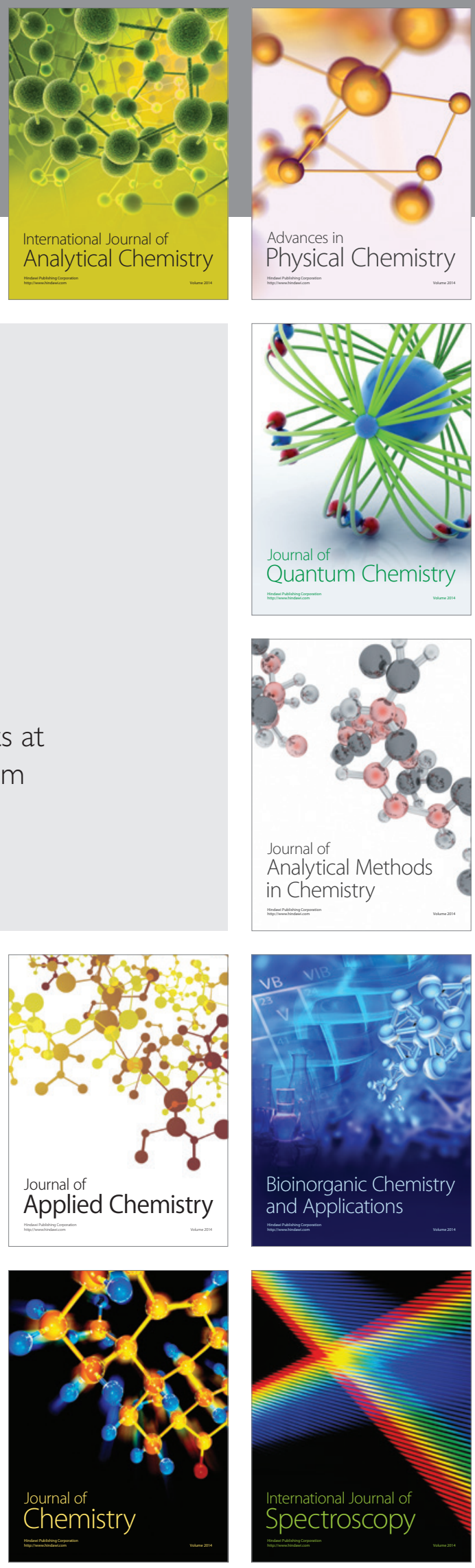